\title{
Rcl1 Suppresses Tumor Progression of Hepatocellular Carcinoma: A Comprehensive Analysis of Bioinformatics and in Vitro Experiments
}

Jiaze Yu

Zhongshan Hospital Fudan University https://orcid.org/0000-0002-3225-1467

Hou Sinan

Zhongshan Hospital Fudan University

Yang Minjie

Zhongshan Hospital Fudan University

Zhou Yongjie

Zhongshan Hospital Fudan University

Du Nan

Zhongshan Hospital Fudan University

Wang Liangwen

Zhongshan Hospital Fudan University

Zhang Wen

Zhongshan Hospital Fudan University

Luo Jianjun

Zhongshan Hospital Fudan University

Yan Zhiping ( $\nabla$ yan.zhiping@zs-hospital.sh.cn )

Zhongshan Hospital Fudan University https://orcid.org/0000-0002-9352-3526

\section{Research Article}

Keywords: Rcl1, HCC, rRNA processing factor, cell cycle, Immune cells infiltration

Posted Date: December 29th, 2021

DOI: https://doi.org/10.21203/rs.3.rs-1124616/v1

License: (c) (1) This work is licensed under a Creative Commons Attribution 4.0 International License.

Read Full License 


\section{Abstract \\ Background}

RNA 3'-terminal phosphate cyclase-like protein (Rcl1) is involved in pre-rRNA processing, but its implication in cancers remains unclear.

\section{Methods}

RCL 1 expressions in 21 malignancies was examinated through GEPIA website portal. Clinical implication data related to RCL 1 level in Hepatocellular Carcinoma (HCC) samples were downloaded through TCGA, ICGC, GEO databases. Survival analysis and gene function enrichment analyses were performed through $\mathrm{R}$ software. The correlation between $R C L 1$ expression and tumor immune infiltration was assessed via the TIMER2.0 database. The effects of Rcl1 overexpression or knockdown on cell growth and metastasis was evaluated by CCK8, transwell, and cell cycle assays.

\section{Results}

$R C L 1$ expression is commonly down-regulated in HCC. The lower expression of $R C L 1$ is associated with higher tumor stage, higher AFP level, vascular invasion, and poor prognosis. RCL 1 expression has a significant correlation with immune cells infiltration in HCC, especially myeloid-derived suppressor cell (MDSC). Moreover, it was further identified that Rcl1 expression was reduced in HCC cell lines and negatively correlated with invasion of HCC cell lines. Immunofluorescence (IF) analysis revealed that the level of Rcl1 expression in the cytoplasm of HCC cells is significantly lower than that in the cytoplasm of L-02 cell. Moreover, both gain- and loss-of-function studies demonstrated that Rcl1 inhibited the growth and metastasis of HCC cells and regulated cell cycle progression in vitro.

\section{Conclusions}

Rcl1 may serve as a novel tumor suppressor in HCC, and its biological effect needs further study.

\section{Highlight}

Rcl1 mRNA expression is down-regulated within HCC tissues and associated with poor prognosis and disease progression. Anti-cancer effects of Rcl1 on HCC were confirmed in vitro. Rcl1 may be a potential tumor suppressor in HCC.

\section{Introduction}


Primary liver cancer, the 6th most prevalent malignancy in the world, is the third leading cause of cancerassociated death worldwide [1]. Hepatocellular carcinoma (HCC) is the most common subtype of primary liver cancer (75-85\%) [2]. Despite the large numbers of effective treatments of mid-advanced HCC exist, the long-term prognosis still remains poor. Therefore, lucubrating on the molecular mechanism of HCC development could contribute to the identification of new therapeutic targets.

Ribosome had initially been considered as a completely homogenous cellular organelle that simply synthesize the protein. However, ribosome heterogeneity was suggested having as evidence the findings that the ribosome proteome, ribosome gene transcriptome, and ribosome biogenesis factors differ between cells and tissues [3, 4]. Besides, the different underlying causes of ribosomopathies and their tissue-specific phenotypes also pointed out the variation in the ribosome composition and function $[5,6]$. Furthermore, many studies have showed that the widespread deficiency in ribosome function and regulation of ribosome activity by oncogenes both could promote cancer development and progression [7]. Furthermore, it was reported that ribosome composition, maturation, and function could promote in the cancer chemo-resistance [8, 9]. In particular, many agents targeted into ribosome could be sensitive to several cancers that had not responded to chemotherapy $[10,11]$. Therefore, studing the role of ribosome in cancer development becomes a necessity.

RCL 1 encodes the RNA 3'-terminal phosphate cyclase-like protein, a number of RNA cyclase families but without cyclase activity [12]. In a yeast study, it was suggested that Rcl1p could serve as an endonuclease that affects the cleavage steps in the $5^{\prime}$-external transcribed spacer and internal transcribed spacer-1 regions of the ribosomal RNA precursor $[13,14]$. However, the endonuclease activity of Rcl1 in human cells is controversial [15]. Minguez et al [16] reported that Rcl1 mRNA expression was associated with vascular invasion of HCC through transcription sequencing. Until now, the role and the exact mechanisms of Rcl1 in HCC development still remains unclear.

In the present study, we explored the mRNA expression and the clinical implications of Rcl1 in HCC patients by using several HCC cohorts. Moreover, the effects and mechanisms of Rcl 1 in HCC cell line was further studied through in vitro experiments.

\section{Materials And Methods}

\section{Data resource}

$R C L 1$ expression in various cancer and its relationship with tumor progression were analyzed via employing the GEPIA2 web portal (http://gepia2.cancer-pku.cn/) and TISIDB web portal (http://cis.hku.hk/TISIDB). Public HCC gene expression matrix were download from Gene Expression Omnibus (GEO) database, Liver Hepatocellular Carcinoma Project of The Cancer Genome Atlas (TCGALIHC), and Liver Cancer - RIKEN, JP Project from International Cancer Genome Consortium (ICGC-LIRI-JP). The correlation of RCL 1 expression with the abundance of immune infiltrates was obtained from the TIMER 2.0 (http://timer.cistrome.org/). The gene sets were downloaded from the Molecular Signatures 
Database (MSigDB) from the Gene Set Enrichment Analysis (GSEA) website (http://www.broadinstitute.org/gsea/msigdb/).

\section{Cell culture}

All human HCC cell lines including Hep-3B, Huh-7, SNU-387, and Li-7, as well as normal human hepatocyte L-02, were obtained from the Cell Bank of the Chinese Academy of Sciences (Shanghai, China). Cells were maintained in Dulbecco's Modified Eagle's medium (DMEM, Hyclone, Logan, UT, USA), Minimum Essential Medium (MEM, Hyclone, Logan, UT, USA), or Roswell Park Memorial Institute-1640 (RPMI-1640, Hyclone, Logan, UT, USA) supplemented with 10\% fetal bovine serum (FBS, LONSERA), streptomycin $(100 \mathrm{mg} / \mathrm{ml})$, and penicillin $(100 \mathrm{unit} / \mathrm{mL})$ at $37^{\circ} \mathrm{C}$ in $5 \% \mathrm{CO}$.

\section{Transient Transfection for Overexpression and Knockdown of Rcl1}

The overexpression vector targeting Rcl1 (FLAG-Rcl1) and a negative control (Ctrl) was conducted with assistance from Genechem CO., Ltd. (Shanghai, China). The Rcl1 short hairpin RNA (shRNA) vector (shRcl1) and its negative control (Ctrl) were also synthesized by Genechem CO., Ltd. (Shanghai, China). For Rcl1 knockdown, shRcl1 targeting the sequence of 5'GCATTGGTTTCTCCAACCTTA3' and the control sequence 5'TTCTCCGAACGTGTACACGT3'. Transfections were performed using lipofectamine 2000 (Invitrogen) according to the manufacturer's protocols.

\section{RNA extraction and quantitative Real Time-polymerase chain reaction (qRT-PCR)}

Total RNA was extracted from HCC cell lines using EZ-press RNA Purification Kit (EZBioscience, China) and then reverse transcribed into cDNA using EZscript Reverse Transcription Mixture (EZBioscience, China) according to the manufacturer's protocol. Quantitative Real-Time PCR was performed using the SYBR Green master mixture (EZBioscience, China) according to the manufacturer's protocol. The following PCR primers were used:

Rcl1 forward: 5' - ATCTGTGGAACATGACTGTAGCG - 3',

Rcl1 reverse: 5' - ATCATTGGTCACTCCTCGTAGA - 3'.

Tubulin forward: 5' - TGGACTCTGTTCGCTCAGGT - 3',

Tubulin reverse: 5' - TGCCTCCTTCCGTACCACAT - 3'.

\section{Western blot (WB)}


Cells were lysed in RIPA buffer (Beyotime Biotechnology, China) containing 1X Protease and Phosphatase inhibitor (Beyotime Biotechnology, China). An equal amount of protein samples was separated by $8 \%$ SDS/PAGE and transferred to $0.25 \mathrm{~mm}$ polyvinylidene fluoride membranes (Millpore, Germany). The membranes were blocked with $5 \%$ non-fat milk for $1 \mathrm{~h}$, then incubated with the individual antibody at $4{ }^{\circ} \mathrm{C}$ overnight: Tubulin (YFB6011, YIFAN BIOLOGICAL), Rcl1 (15330-1-AP, Proteintech), GAPDH (bs-0755R, Bioss). The membranes were then incubated with the second antibody (bs-40295G-HRP, Bioss) at $37^{\circ} \mathrm{C}$ for $1 \mathrm{~h}$. Finally, protein bands were visualized using Omni ECL reagent (EpiZyme, China), and the gray intensity was acquired by using Fiji (NCBI, USA).

\section{Cell proliferation assay}

Cell viability was monitored by Cell Count Kit 8 (Absin, China) according to the manufacturer's protocol. Briefly, HCC cells were counted and plated onto 96 -well cell culture plates at a density of $2 \times 10^{3} /$ well. Proliferation rates were measured by absorbance of $450 \mathrm{~nm}$ at $0,1,2,3$, and 4 days post-transfection. Experiments were repeated more than three times with similar results.

\section{Immunofluorescence}

HCC cells were seeded on a $35 \times 35 \mathrm{~mm}$ confocal cell culture plate (Thermal, USA). After fixed using $4 \%$ paraformaldehyde, cultured cells were blocked with Immunol staining blocking buffer (Beyotime, China) with $0.3 \%$ Triton X-100 (Beyotine, China) in PBS for 30 min at RT. The samples were then incubated with primary antibody overnight at $4^{\circ} \mathrm{C}$ : Rcl1 (15330-1-AP, Proteintech), followed by the appropriate secondary fluorescently labeled antibodies (Invitrogen, USA) for 1 hour at $37^{\circ} \mathrm{C}$. Nuclei were counterstained with DAPI (Beyotime). Images were analyzed by a laser scanning confocal microscope (Olympus, FV300).

\section{Transwell assay}

For migration assay, $\mathrm{HCC}$ cells were seeded $\left(7 \times 10^{4}\right)$ onto the upper chamber wells with serum-free medium and then incubating for $48 \mathrm{~h}$. The penetrated cells were fixed with $4 \%$ paraformaldehyde and stained with crystal violet. For the Matrigel invasion assay, the 24-well transwell chamber was coated with an extracellular matrix on the upper surface (Corning, USA). Then, HCC cells were seeded $\left(1.5 \times 10^{5}\right)$ onto the upper chamber wells with serum-free medium. After incubating for $48 \mathrm{~h}$, the penetrated cells were fixed with $4 \%$ paraformaldehyde and stained with crystal violet. Then, migrating or invading cells were photographed at $100 \times$ and counted in five random fields.

\section{Cell cycle analysis}

The cell cycle was analyzed by Cell Cycle Assay Kit Plus (US EVERYBRIGHT INC, China) according to the protocol. Briefly, HCC cells were collected and washed twice in cold phosphate-buffered saline (PBS), and 
then fixed in $70 \%$ cold ethanol at $-20^{\circ} \mathrm{C}$ for $24 \mathrm{~h}$. The samples were washed twice in cold staining buffer and resuspended in $1 \mathrm{~mL}$ PBS with $4 \mathrm{ul}$ RedNucleus I staining solution. After incubation for $20 \mathrm{~min}$ at RT in the dark, the cell cycle was evaluated by flow cytometry (BD bioscience, USA). The ModFit LT5.0 (Verify Software House, USA) was used to analyze the cell cycle.

\section{Statistical analysis}

Statistical analyses were performed using R software (version 4.0.0, USA) or GraphPad Prism (La Jolla, USA). The limma package was used to evaluate Rcl1 expression between HCC tumor tissues and adjacent tissues using R software. The survival data of HCC patients were analyzed via log-rank test and Cox proportional hazard regression. According to the relevance of Rc/1 expression, genes in TCGA-LIHC were devided into Rcl $1^{\text {positive }}$ and Rcl $1^{\text {negative }}$ subgroups. Gene set enrichment analysis (GSEA) was performed using $\mathrm{R}$ software. Continuous data were expressed as the mean \pm standard deviation (SD). Comparisons between groups were performed using Student's $t$ test or ANOVA test. A value of $p<0.05$ was defined as statistically significant.

\section{Results}

\section{$R C L 1$ is abnormally expressed in various tumor tissues, and associated with prognosis and tumor progression.}

We first explored the general expression of $R c / 1$ in multiple human cancers using the GEPIA2 website portal (Figure 1A). The analyses of the RNA-seq data of 23 malignancies in TCGA suggested that the expression of RCL 1 was significantly lower in Cholangiocarcinoma and LIHC compared to the adjacent normal tissues. However, RCL 1 expression of Colon and Rectum adenocarcinomas were significantly higher than the one in normal tissues.

The TISIDB web portal was used (Figure 1B) to further evaluate the correlation of $R C L 1$ expression and survival prognosis. Notably, RCL 1 expression had a significant impact in the prognosis of 8 cancers, including brain lower-grade glioma (LGG), glioblastoma multiforme, kidney renal clear cell carcinoma (KIRC), LIHC, ovarian serous cystadenocarcinoma(OV), uterine corpus endometrial carcinoma (UCEC), uterine carcinosarcoma (UCS), and uveal melanoma (UVM). Low RCL 1 expression was remarkably associated with poor prognosis in all these cancer types except for UCEC and UCS.

Moreover, the association between $R C L 1$ expression and tumor progression across human cancers was identified. It was revealed that $R C L 1$ expression was positively correlated to tumor stage in KIRC, LIHC, and Stomach adenocarcinoma, as well as in UVM ((Figure 1C). Similarly, the expression of RCL 1 in KIRC and LIHC was also positively associated with histological grade ((Figure 1D). However, the RCL 1 downregulation was notably correlated with higher grade of Cervical squamous cell carcinoma and endocervical adenocarcinoma and UCEC (Figure 1D). 
In summary, these results confirmed that RCL1 could be a potential tumor-associated gene in several melignancies. Notably, it was in LIHC that $R C L 1$ expression was not only significantly down-regulated but also associated with prognosis, tumor progression across many human cancers.

\section{Low RCL1 expression is correlated with poor clinicpathological outcomes in HCC patients.}

Eleven HCC datasets were downloaded and analyzed (Figure 1E) to further verify RCL 1 expression in HCC tumor tissue. For 9 HCC datasets including GSE22058, GSE25097, GSE36376, GSE14520, TCGALIHC, GSE76427, GSE54236, GSE63898, and ICGC-LIR-JP, the expression levels of Rc/1 in HCC tissues were generally lower than the ones in adjacent tissues $(p<0.001)$.

Univariate and multiple survival analyses were performed using $\mathrm{R}$ programming environment on the TCGA-LIHC dataset to promote understanding of the association between the RCL 1 expression and the prognosis of HCC. Univariate analysis indicated that patients with the high RCL 1 expression was associated with better overall survival (OS, HR $=0.607(0.416-0.886))$ and progression-free survival (PFS, $\mathrm{HR}=0.661$ (0.476 - 0.917)) (Figure 2A-2B). Univariate and multivariate analyses further confirmed that $R C L 1$ expression is an independent factor for OS $(\mathrm{HR}=0.616(0.420-0.905))$ and PFS $(\mathrm{HR}=0.701$ $(0.502-0.98)$ ) of HCC patients (Figure 2C, D, Table 1).

In addition, we studied the connection between $R C L 1$ expression and the clinicopathological characteristics. It was found that low RCL 1 expression was significantly correlated with female, advanced primary tumor (T classification) and TNM stage, higher AFP level, as well as vascular invasion in TCGALIHC cohort (Figure 3A-E). Besides, it was found that a decrease in the RCL 1 expression was associated with increasing T classification, HBV infection, portal vein and hepatic vein invasion in ICJC-LIRI-JP corhort (Figure 3F-I). Meanwhile, a remarkable connection between RCL 1 expression was lower in the patients with BCLC C stage, proliferation class, high AFP level, vascular invasion, as well as phosphorylation level of Akt, RPS6, and IGFR1 in GSE9843 corhort (Figure 3J-P). No significant relationship between the RCL1 mRNA expression and age, cirrhosis, as well as gender was found in HCC cohorts (Figure S1).

\section{$R C L 1$ expression significantly correlates with infiltrating levels of various immune cells in $\mathrm{HCC}$, especially $\mathrm{CD} 4^{+} \mathrm{T}$ cells.}

The correlation of $R C L 1$ expression in HCC samples with immune infiltration levels were investigated using TIMER2.0 website portal. The results showed that RCL 1 expression significantly correlated with the infiltrating levels of myeloid-derived suppressor cell (MDSC, $r=-0.395, p=2.32 \mathrm{e}-14)$, endothelial cell $(r=$ $0.336, p=1.57 \mathrm{e}-10)$, hematopoietic stem cell $(r=0.296, p=2.21 \mathrm{e}-08)$, Tregs $(r=0.279, p=1.34 \mathrm{e}-07)$, monocyte $(r=0.261, p=9.2 \mathrm{e}-07)$, granulocyte-monocyte progenitor $(r=0.255, p=1.53 \mathrm{e}-06), \mathrm{CD} 4+\mathrm{T}$ cells $(r=-0.31, p=3.92 \mathrm{e}-09), \mathrm{DCs}(r=-0.204, p=1.35 \mathrm{e}-04)$, macrophages $(r=-0.197, p=2.35 \mathrm{e}-04), \mathrm{CD} 8+\mathrm{T}$ 
cells $(r=0.16, p=2.81 \mathrm{e}-03)$, B cells $(r=-0.125, p=2.01 \mathrm{e}-02)$, and neutrophils $(r=-0.133, p=1.36 \mathrm{e}-02)$ in $\mathrm{LIHC}$, although no significant correlation with tumor purity was found (Figure 4). Furthermore, the RCL 1 expression was negatively associated with the infiltration level of naïve CD4+ T cell $(r=-0.131, p=1.51 \mathrm{e}-$ 02), Th1 cell $(r=-0.182, p=6.78 \mathrm{e}-04)$, Th2 cell $(r=-0.245, p=3.98 \mathrm{e}-06)$, M0 macrophages $(r=-0.266, p=$ $5.44 \mathrm{e}-07)$, but not with M1 macrophages $(r=0.025, p=6.40 \mathrm{e}-01)$ and $\mathrm{M} 2$ macrophages $(r=-0.081, p=$ 1.33e-01) (Figure 4).

Moreover, $R C L 1$ expression was found to be significantly different between molecular and immune subtypes by exploring the TISIDB web portal $(p<0.001$, Figure 5A-B). Furthermore, the expression levels of $R C L 1$ in the patients with TP53 and IDH1 mutation were lower than the ones in the patients with TP53 and IDH1 wild-type (Figure 5C, 5D). The expression levels of $R C L 1$ in the patients with CTNNB1 mutation were higher than the ones in the patients with CTNNB1 wild-type (Figure 5E) and no significant difference was observed between the expression levels of RCL 1 in TERT mutation and wild-type patients (Figure 5F).

\section{Rcl1 is down expression in HCC cells and suppresses HCC cell growth and metastasis in vitro.}

The endogenous Rcl1 expression levels were detected in a collection of liver cancer cell lines and L-02 cells. Both mRNA and protein expression levels of Rcl1 were generally lower in all liver cancer lines in comparison with L-02 cells (Figure 6A, 6B). The Rcl1 expression of high-invasive HCC cell lines was substantially lower than the one in the low-invasive cell lines. These findings were further supported by immunofluorescence staining (Figure 6C). Interestingly, it was also revealed that there was a marked difference in the distribution of Rcl1 protein between the liver cells and cancer cells. In particular, Rcl1 was mostly located in the nucleus in the HCC cell lines, while it was uniformly distributed in nucleus and cytoplasm in the liver cell lines (Figure 6C).

Then a recombinant plasmid vector encoding Rcl1 (FLAG-Rcl1) was conducted and an empty vector was used as control (Ctrl). The overexpression of Rcl1 in Huh-7 cells was validated with RT-PCR and western blot analyses (Figure 7A, 7C). Forced Rcl1 expression could markedly inhibit cell growth as supported by cell viability assay in Huh-7 cell (Figure 7E). Moreover, transwell assays indicated that the overexpression of Rcl1 significantly impaired Huh-7 cell's ability to migrate and invade (Figurre 7G). Furthermore, Rcl1 was knockdown by transfecting the shRcl1 vector or empty vector into Hep-3B cell line. The efficiency of knockdown was confirmed by RT-PCR and western blot analyses (Figure 7B, 7D). Consistently, knockdown of Rcl1 in Hep-3B cell strikingly enhanced cell viability, migration, and invasion (Figure 7F, 7H).

\section{Rcl1 could potentially participate in regulating cell cycle and metabolism-associated signal pathways.}


Notably, the Rcl $1^{\text {positive }}$ group of genes were enriched in multiple cellular metabolic processes such as xenobiotic, fatty acid, bile acid, adipogenesis, and oxidative phosphorylation, while the Rcl 1 negative subgroup of genes were enriched in cell cycle regulation including G2M checkpoint, E2F targets and mitotic spindle (Figure 8A, C). Moreover, gene ontology (GO) analysis revealed that Rcl1 could potentially promote the activation of protein-binding and transmembrane transport, while simultaneously inhibiting microtubule and protein kinase activity (Figure 8B). Mitochondria and chromosomes were the main cellular organelles of the Rcl $1^{\text {positive }}$ and Rcl ${ }^{\text {negative }}$ groups, respectively (Figure 8D).

The effects of Rcl1 on cell cycle distribution was evaluated using flow cytometry analysis. We found that the overexpression of Rcl1 resulted in a significant increase of cells in $\mathrm{G} 2 \mathrm{M}$ phase $(35.10 \%$ vs $22.06 \%, p=$ $0.002)$ and a concomitantly significant decrease of cells in S phase $(25.62 \%$ vs $39.53 \%, p=0.0002)$ in Huh-7 cell (Figure 8E). As expected, upon Rcl1 knockdown, the ratio of cells in G2M phase was decreased (18.89\% VS 26.58\%, $p=0.003)$, the ratio in S phase was increased (32.33\% VS 17.59\%, $p=0.0001)$, and the ratio in the $\mathrm{G} 0 / 1$ phase was decreased $(50.78 \%$ VS $55.84 \%, p=0.040)$ (Figure $7 \mathrm{~F}$ ).

\section{Discussion}

Rcl1 could interact with Bms1 to endonucleolytic cleave the pre-rRNA at the A2 site, which is required for the eukaryotic pre-ribosome assembly implicated in the 18s rRNA biogenesis [17-19]. However, the mechanism that allows Rcl1 to carry out the catalytic activity in human species still remains unclear since the mutation of the proposed pre-rRNA sub substrate binding site of human Rcl1 does not affect 2a cleavage or $18 \mathrm{~S}$ production [15]. In the present study, we found that the expression levels of $R C L 1$ was primarily reduced in HCC tissues and cell lines. Moreover, its expression significantly correlated with the survival prognosis, tumor progression, immune infiltration level, as well as molecular classification of HCC. Furthermore, both gain- and loss-of-function experiments demonstrated that Rcl1 has the inhibitory effects on the proliferation, migration, and invasion of HCC cells in vitro. Bioinformatic analyses revealed that $R c / 1$ could be significantly associated with cell cycle transition, cellular metabolic processes. Mechanistically, it was identified that Rcl1 overexpression or knockdown could inhibit or promote cell cycle transtion.

$R C L 1$ was found to be up-regulated in colorectal cancers, but downregulated in liver and bile duct cancers. Venkateswaran et al. found that non-coding variants of RCL 1 could regulate granulocytemacrophage colony-stimulating factor signaling in inflammatory bowel disease patients [20]. It is likely that single nucleotide polymorphism of RCL 1 influences the gut microbiome. Gut microbiome has a key role in carcinogenesis and anticancer immune response of HCC and colorectal cancer [21,22]. And low $R C L 1$ expression was associated with poor prognosis and tumor stage in multiple cancers. It suggested that Rcl1 may play a dualistic role by acting both as an oncogene and tumor suppressor, which is similar to other ribosome biogenesis factors [23].

Many studies have elucidated that many ribosome assembly factors could promote cell growth and metastasis in HCC by up-regulating the rate of ribosome biogenesis [9, 24-28]. But we found that RCL 1 
expression was commonly downregulated in HCC tissues and cell lines. Besides, bioinfomatic analyses indicated that low RCL 1 expression was th e risk factor of poor survival prognosis and tumor progression, including: advanced TNM classification, high AFP level, vascular invasion in many HCC corhorts. And RCL 1 expression was relatively lowest in iCluster 1 and proliferation molecular subtypes of HCC which exhibited the high frequence of macrovascular invasion and a significantly worse prognosis [29].

Recently, Zhu et al. reported that Rcl1 is essential for the 18S rRNA maturation at A1-site and for digestive organogenesis in zebrafish [30]. And its deficiency may upregulate the expression of genes responsible for ribosome biogenesis. Meanwhile, we also found that Rcl1 protein was distributed in both nucleus and cytoplasm of HCC cell and hepatocyte lines by immunofluorescence, which is consistent with a recent study [31]. Notably, the cytoplasmic Rcl1 protein level of HCC cell lines were dramatically reduced compared to hepatocyte cell. And it has been documented that the localization of BOP1, one of ribosome biogenesis factors, from nucleus to cytoplasm correlated with advanced disease and decreased survival in prostate cancer patients. Further research is needed to be proven whether Rcl1 could participate in the biological processes in the cytoplasm.

Moreover, functional tests indicated Rcl1 may be a potential tumor suppressor in HCC in vitro. Mechanisticlly, GSEA also suggested RCL1 was involved in cell cycle control and multiple cellular metabolic processes. It was further identified that Rcl1 overexpression could induce HCC cell cycle arrest by flow cytometer analysis. Consistently, Rcl1 knockdown obviously promote cell cycle progression of HCC cell. Large number of studies have demonstrated that aberrant pre-rRNA intermediates could lead to cell cycle arrest [32-35]. The most widely accepted model of cell-cycle arrest, upon pre-rRNA processing defect, was that the PeBoW complex could increase the extra-ribosome RPL5-RPL11-5S rRNA complex to bind and block the human homolog of mouse double minute-mediated ubiquitination and degradation of p53 [34, 36-38]. But it was documented that exogenous expression of Rcl1 in senescent cells could not increase the accumulation of p53, p21, and p16 [39]. Combining the bioinformatic analyses and flow cytometer analyasis, we found that Rcl1 mainly control cell cycle progression by interfering the $S$ phase.

Immune-related mechanisms play notable roles in the incidence and recurrence of $\mathrm{HCC}$, and a combination of molecular and immune therapies could remarkably increase objective response in advanced HCC by $30-40 \%$ [40]. Ribosome biogenesis modulate immunosurveillance and innate immune response [41-43]. We also examined the correlation between the RCL1 expression and the infiltration levels of several immune cells in the TCGA-LIHC corhort. There exist a moderate negative relationship between the Rc/1 expression and the infiltration level of MDSC, CD4+ T cell, and Tregs, and significantly positive correlations with the infiltration level of endothelial cell, hematopoietic stem cell, and CD8+ T cells. MDSC contributes to the immunosuppressive network through multiple mechanisms and mediates the tumor growth, angiogenesis, and metastasis of HCC [44] while endothelial cell necroptosis induced by tumor-cells could reversely promote cancer metastasis [45]. Moreover, we also found a prominent association between the expression levels of $R C L 1$ and the immune-subtypes in HCC patients. The RCL 1 expression was relatively lower in the $\mathrm{C} 1$ subtype (wound healing). Its characteritics include a high proliferation rate, the upregulated expression of angiogenic genes, a Th2 cell-dominated immune infiltration, as well as less favorable outcomes. Recently, Jung et al found that an rRNA fragment 
containing 2',3'-cyclic phosphate and guanosine triphosphate (GTP) -binding activity functions as an endogenous RIG-I ligand to induce immune stimulation [46]. It is generally accepted that the interaction between Rcl1 and Bms1, a GTPase-activating protein, is involved in pre-rRNA processing across species $[17,18]$. Further observational studies are required to confirm whether the Rcl1-Bms1 complex could module the innate stimulation by activating RIG-I.

\section{Conclusions}

In summary, our study demonstrates that Rcl1 could serve as a favourable prognostic factor for HCC. Moreover, intracellular molecular metabolism and cell cycle control might be the paimary biological processes regulated by Rcl1. Furthermore, the results of cell experiment indicated that Rcl1 plays a pivotal anti-cancer role by inhibitung of both growth and metastasis of HCC. The significant reduction of cytoplasmic Rcl1 protein in HCC imply the additional biological function. Our study revealed that Rcl1 may act as a potential prognostic marker and tumor supressor in HCC.

\section{Abbreviations}

DMEM Dulbecco's Modified Eagle's medium

EMT epithelial mesenchymal transition

FBS fetal bovine serum

GO gene ontology

GSEA Gene set enrichment analysis

GTP guanosine triphosphate

KIRC kidney renal clear cell carcinoma

LGG brain lower-grade glioma

LIHC Liver hepatic carcinoma

MEM Modified Eagle's medium

OS overall survival

PFS progression free survival

qRT-PCR quantitative real time-polymerase chain reaction

Rcl1 RNA 3'-terminal phosphate cyclase-like protein 
RPMI-1640 Roswell Park Memorial Institute-1640

UCES Uterine corpus endometrial carcinoma

UCS Uterine carcinosarcoma

UVM Uveal melanoma

WB western blot

\section{Declarations}

\section{Acknowledgements}

We would like to thank the researchers for their contributions, and express our gratitude to EditSprings (https://www.editsprings.com/) for the expert linguistic services provided. We also gratefully acknowledge TCGA, GEPIA, TISIDB, GSEA for providing data to analyze.

\section{Authors' contributions}

Conceptualization, YJZ, ZW, LJJ and YZP, methodology, YJZ, HSN, software, YJZ, HSN, YMJ, ZYJ, DN, WLW, validation, YJZ, ZW, LJJ and YZP, formal analysis, YJZ, HSN, YMJ, ZYJ, DN, WLW, investigation, YJZ, HSN, resources, YJZ, HSN, YMJ, ZYJ, DN, WLW, data curation, YJZ, HSN, YMJ, ZYJ, DN, original draft preparation, YJZ, HSN, review and editing, ZW, visualization, ZW, LJJ, supervision, YZP, project administration, YZP, funding acquisition, YZP. All authors have read and agreed to the published version of the manuscript.

\section{Funding}

This research was funded by National Natural Science Foundation of China, grant number 81771948 , and the funding from Zhongshan Hospital, grant number $2016 Z$ SLC17.

\section{Availability of data and materials}

The RNA-sequencing data and corresponding clinical information were downloaded from The Cancer Genome Atlas (TCGA) database (https://portal.gdc.cancer.gov/), International Cancer Genome Consortium (ICGC) databases (https://dcc.icgc.org/), Gene Expression Omnibus (GEO) databases (https://www.ncbi.nlm.nih.gov/geo/).

\section{Competing interests}

The authors declare no conflict of interest.

\section{Consent for publication}


Not applicable.

Ethics approval and consent to participate

Not applicable.

\section{References}

1. Sung H, Ferlay J, Siegel RL, Laversanne M, Soerjomataram I, Jemal A, Bray F: Global cancer statistics 2020: GLOBOCAN estimates of incidence and mortality worldwide for 36 cancers in 185 countries. CA Cancer J Clin 2021.

2. Llovet JM, Kelley RK, Villanueva A, Singal AG, Pikarsky E, Roayaie S, Lencioni R, Koike K, ZucmanRossi J, Finn RS: Hepatocellular carcinoma. Nat Rev Dis Primers 2021, 7(1):6.

3. Ford D: Ribosomal heterogeneity - A new inroad for pharmacological innovation. Biochem Pharmacol 2020, 175:113874.

4. Shi Z, Fujii K, Kovary KM, Genuth NR, Rost HL, Teruel MN, Barna M: Heterogeneous Ribosomes Preferentially Translate Distinct Subpools of mRNAs Genome-wide. Mol Cell 2017, 67(1):71-83.e77.

5. Genuth NR, Barna M: The Discovery of Ribosome Heterogeneity and Its Implications for Gene Regulation and Organismal Life. Mol Cell 2018, 71(3):364-374.

6. Bohnsack KE, Bohnsack MT: Uncovering the assembly pathway of human ribosomes and its emerging links to disease. EMBO J 2019, 38(13).

7. Pelletier J, Thomas G, Volarević S: Ribosome biogenesis in cancer: new players and therapeutic avenues. Nat Rev Cancer 2018, 18(1):51-63.

8. Chen ZH, Qi JJ, Wu QN, Lu JH, Liu ZX, Wang Y, Hu PS, Li T, Lin JF, Wu XY et al: Eukaryotic initiation factor 4A2 promotes experimental metastasis and oxaliplatin resistance in colorectal cancer. $J$ Exp Clin Cancer Res 2019, 38(1):196.

9. Ruan Y, Sun L, Hao Y, Wang L, Xu J, Zhang W, Xie J, Guo L, Zhou L, Yun X et al: Ribosomal RACK1 promotes chemoresistance and growth in human hepatocellular carcinoma. The Journal of clinical investigation 2012, 122(7):2554-2566.

10. Kearse MG, Goldman DH, Choi J, Nwaezeapu C, Liang D, Green KM, Goldstrohm AC, Todd PK, Green $\mathrm{R}$, Wilusz JE: Ribosome queuing enables non-AUG translation to be resistant to multiple protein synthesis inhibitors. Genes Dev 2019, 33(13-14):871-885.

11. Lawrence MG, Obinata D, Sandhu S, Selth LA, Wong SQ, Porter LH, Lister N, Pook D, Pezaro CJ, Goode DL et al: Patient-derived Models of Abiraterone- and Enzalutamide-resistant Prostate Cancer Reveal Sensitivity to Ribosome-directed Therapy. Eur Urol 2018, 74(5):562-572.

12. Billy E, Wegierski T, Nasr F, Filipowicz W: Rcl1p, the yeast protein similar to the RNA 3'-phosphate cyclase, associates with U3 snoRNP and is required for 18S rRNA biogenesis. EMBO J 2000, 19(9):2115-2126. 
13. Horn DM, Mason SL, Karbstein K: Rcl1 protein, a novel nuclease for $18 \mathrm{~S}$ ribosomal RNA production. J Biol Chem 2011, 286(39):34082-34087.

14. Tanaka N, Smith P, Shuman S: Crystal structure of Rcl1, an essential component of the eukaryal prerRNA processosome implicated in 18s rRNA biogenesis. RNA 2011, 17(4):595-602.

15. Wells GR, Weichmann F, Colvin D, Sloan KE, Kudla G, Tollervey D, Watkins NJ, Schneider C: The PIN domain endonuclease Utp24 cleaves pre-ribosomal RNA at two coupled sites in yeast and humans. Nucleic Acids Res 2016, 44(11):5399-5409.

16. Minguez B, Hoshida Y, Villanueva A, Toffanin S, Cabellos L, Thung S, Mandeli J, Sia D, April C, Fan JB et al: Geneexpression signature of vascular invasion in hepatocellular carcinoma. J Hepato/ 2011, 55(6):1325-1331.

17. Delprato A, Al Kadri Y, Pérébaskine N, Monfoulet C, Henry Y, Henras AK, Fribourg S: Crucial role of the Rcl1p-Bms1p interaction for yeast pre-ribosomal RNA processing. Nucleic Acids Res 2014, 42(15):10161-10172.

18. Wang Y, Zhu Q, Huang L, Zhu Y, Chen J, Peng J, Lo LJ: Interaction between Bms1 and Rcl1, two ribosome biogenesis factors, is evolutionally conserved in zebrafish and human. J Genet Genomics 2016, 43(7):467-469.

19. Tafforeau L, Zorbas C, Langhendries J-L, Mullineux S-T, Stamatopoulou V, Mullier R, Wacheul L, Lafontaine Denis LJ: The Complexity of Human Ribosome Biogenesis Revealed by Systematic Nucleolar Screening of Pre-rRNA Processing Factors. Mol Cell 2013, 51(4):539-551.

20. Venkateswaran S, Denson LA, Jurickova I, Dodd A, Zwick ME, Cutler DJ, Kugathasan S, Okou DT: Neutrophil GM-CSF signaling in inflammatory bowel disease patients is influenced by non-coding genetic variants. Sci Rep 2019, 9(1):9168.

21. Song M, Chan AT, Sun J: Influence of the Gut Microbiome, Diet, and Environment on Risk of Colorectal Cancer. Gastroenterology 2020, 158(2):322-340.

22. Schwabe RF, Greten TF: Gut microbiome in HCC - Mechanisms, diagnosis and therapy. $J$ Hepatol 2020, 72(2):230-238.

23. Srinivas AN, Suresh D, Mirshahi F, Santhekadur PK, Sanyal AJ, Kumar DP: Emerging roles of AATF: Checkpoint signaling and beyond. J Cell Physio/ 2021, 236(5):3383-3395.

24. Wang J, Sun J, Zhang N, Yang R, Li H, Zhang Y, Chen K, Kong D: PES1 enhances proliferation and tumorigenesis in hepatocellular carcinoma via the PI3K/AKT pathway. Life Sci 2019, 219:182-189.

25. Wang H, Xiao W, Zhou Q, Chen Y, Yang S, Sheng J, Yin Y, Fan J, Zhou J: Bystin-like protein is upregulated in hepatocellular carcinoma and required for nucleologenesis in cancer cell proliferation. Cell Res 2009, 19(10):1150-1164.

26. Yin Y, Zhou L, Zhan R, Zhang Q, Li M: Identification of WDR12 as a novel oncogene involved in hepatocellular carcinoma propagation. Cancer Manag Res 2018, 10:3985-3993.

27. Zhang X, Chen J, Jiang S, He S, Bai Y, Zhu L, Ma R, Liang X: N-Acetyltransferase 10 Enhances Doxorubicin Resistance in Human Hepatocellular Carcinoma Cell Lines by Promoting the Epithelialto-Mesenchymal Transition. Oxid Med Cell Longev 2019, 2019:7561879. 
28. Chung K-Y, Cheng IKC, Ching AKK, Chu J-H, Lai PBS, Wong N: Block of proliferation 1 (BOP1) plays an oncogenic role in hepatocellular carcinoma by promoting epithelial-to-mesenchymal transition. Hepatology 2011, 54(1):307-318.

29. Cancer Genome Atlas Research Network. Electronic address wbe, Cancer Genome Atlas Research N: Comprehensive and Integrative Genomic Characterization of Hepatocellular Carcinoma. Cel/ 2017, 169(7):1327-1341.

30. Zhu Q, Tao B, Chen H, Shi H, Huang L, Chen J, Hu M, Lo LJ, Peng J: Rcl1 depletion impairs 18 S prerRNA processing at the A1-site and up-regulates a cohort of ribosome biogenesis genes in zebrafish. Nucleic Acids Res 2021, 49(10):5743-5759.

31. Brownstein CA, Smith RS, Rodan LH, Gorman MP, Hojlo MA, Garvey EA, Li J, Cabral K, Bowen JJ, Rao AS et al: RCL1 copy number variants are associated with a range of neuropsychiatric phenotypes. Mol Psychiatry 2021.

32. Bernstein KA, Bleichert F, Bean JM, Cross FR, Baserga SJ: Ribosome biogenesis is sensed at the Start cell cycle checkpoint. Mol Biol Cell 2007, 18(3):953-964.

33. Iwanami N, Higuchi T, Sasano Y, Fujiwara T, Hoa VQ, Okada M, Talukder SR, Kunimatsu S, Li J, Saito F et al: WDR55 is a nucleolar modulator of ribosomal RNA synthesis, cell cycle progression, and teleost organ development. PLoS Genet 2008, 4(8):e1000171.

34. Strezoska Z, Pestov DG, Lau LF: Functional inactivation of the mouse nucleolar protein Bop1 inhibits multiple steps in pre-rRNA processing and blocks cell cycle progression. J Biol Chem 2002, 277(33):29617-29625.

35. Pestov DG, Strezoska Z, Lau LF: Evidence of p53-dependent cross-talk between ribosome biogenesis and the cell cycle: effects of nucleolar protein Bop1 on G(1)/S transition. Mol Cell Biol2001, 21(13):4246-4255.

36. Rohrmoser M, Hölzel M, Grimm T, Malamoussi A, Harasim T, Orban M, Pfisterer I, Gruber-Eber A, Kremmer E, Eick D: Interdependence of Pes1, Bop1, and WDR12 controls nucleolar localization and assembly of the PeBoW complex required for maturation of the $60 \mathrm{~S}$ ribosomal subunit. Mol Cell Biol 2007, 27(10):3682-3694.

37. Grimm T, Hölzel M, Rohrmoser M, Harasim T, Malamoussi A, Gruber-Eber A, Kremmer E, Eick D: Dominant-negative Pes1 mutants inhibit ribosomal RNA processing and cell proliferation via incorporation into the PeBoW-complex. Nucleic Acids Res 2006, 34(10):3030-3043.

38. Hölzel M, Rohrmoser M, Schlee M, Grimm T, Harasim T, Malamoussi A, Gruber-Eber A, Kremmer E, Hiddemann W, Bornkamm GW et al: Mammalian WDR12 is a novel member of the Pes1-Bop1 complex and is required for ribosome biogenesis and cell proliferation. J Cell Bio/ 2005, 170(3):367378.

39. Nishimura K, Kumazawa T, Kuroda T, Katagiri N, Tsuchiya M, Goto N, Furumai R, Murayama A, Yanagisawa J, Kimura K: Perturbation of ribosome biogenesis drives cells into senescence through 5S RNP-mediated p53 activation. Cell Rep 2015, 10(8):1310-1323. 
40. Llovet JM, De Baere T, Kulik L, Haber PK, Greten TF, Meyer T, Lencioni R: Locoregional therapies in the era of molecular and immune treatments for hepatocellular carcinoma. Nat Rev Gastroenterol Hepatol 2021.

41. Bianco C, Mohr I: Ribosome biogenesis restricts innate immune responses to virus infection and DNA. Elife 2019, 8.

42. Dersh D, Holly J, Yewdell JW: A few good peptides: MHC class I-based cancer immunosurveillance and immunoevasion. Nat Rev Immunol 2021, 21(2):116-128.

43. Wei J, Kishton RJ, Angel M, Conn CS, Dalla-Venezia N, Marcel V, Vincent A, Catez F, Ferré S, Ayadi L et al: Ribosomal Proteins Regulate MHC Class I Peptide Generation for Immunosurveillance. Mol Cell 2019, 73(6).

44. Lu C, Rong D, Zhang B, Zheng W, Wang X, Chen Z, Tang W: Current perspectives on the immunosuppressive tumor microenvironment in hepatocellular carcinoma: challenges and opportunities. Mol Cancer 2019, 18(1):130.

45. Strilic B, Yang L, Albarran-Juarez J, Wachsmuth L, Han K, Muller UC, Pasparakis M, Offermanns S: Tumour-cell-induced endothelial cell necroptosis via death receptor 6 promotes metastasis. Nature 2016, 536(7615):215-218.

46. Jung S, von Thülen T, Yang I, Laukemper V, Rupf B, Janga H, Panagiotidis G-D, Schoen A, Nicolai M, Schulte $L N$ et al: A ribosomal RNA fragment with 2',3'-cyclic phosphate and GTP-binding activity acts as RIG-I ligand. Nucleic Acids Res 2020.

\section{Tables}

Table 1 Univariate analysis of TCGA-LIHC patient overall survival and progression-free survival.

\begin{tabular}{|lllllll|}
\hline & \multicolumn{2}{l}{ Overall Survival } & & \multicolumn{2}{l|}{ Progression-free Survival } \\
\hline Parameters & HR & $95 \% \mathrm{Cl}$ & $P$ value & HR & $95 \% \mathrm{Cl}$ & $P$ value \\
\hline Age & 1.070 & $0.717-1.597$ & 0.741 & 0.909 & $0.645-1.281$ & 0.585 \\
\hline Gender & 0.750 & $0.511-1.100$ & 0.14 & 0.957 & $0.674-1.36$ & 0.808 \\
\hline HBV & 0.470 & $0.280-0.791$ & $\mathbf{0 . 0 0 4}$ & 0.734 & $0.498-1.082$ & 0.118 \\
\hline HCV & 1.134 & $0.590-2.179$ & 0.707 & 1.703 & $1.048-2.766$ & $\mathbf{0 . 0 3 2}$ \\
\hline Etiology & 1.442 & $0.693-1.821$ & $\mathbf{0 . 0 0 2}$ & 1.035 & $0.846-1.265$ & 0.74 \\
\hline AJCC_T & 2.369 & $1.617-3.471$ & $\mathbf{0 . 0 0 0}$ & 2.242 & $1.582-3.178$ & $\mathbf{0 . 0 0 0}$ \\
\hline Stage & 2.348 & $1.605-3.436$ & $\mathbf{0 . 0 0 0}$ & 2.257 & $1.598-3.188$ & $\mathbf{0 . 0 0 0}$ \\
\hline RCL1 & 0.607 & $0.416-0.886$ & $\mathbf{0 . 0 0 9}$ & 0.661 & $0.476-0.917$ & $\mathbf{0 . 0 1}$ \\
\hline
\end{tabular}

TNM tumor-nodes-metastases; 
AJCC American Joint Committee on Cancer.

Statistically significant $P$ value $(p<0.05)$ were bold processed.

\section{Figures}

\section{Figure 1}

$R C L 1$ is significantly differentially expressed in various tumor tissues, and associated with prognosis and tumor progression. (A) Human RCL 1 expression levels in different tumors and adjacent normal tissues from TCGA database. (B) Correlation between the expression of RCL 1 and the overall survival across human cancers. Longer (or Shorter): the gene is associated with longer (shorter) survival (Log rank test: $p$ $<0.05)$, NS: No significant. (C) Correlation between the expression levels of RCL 1 and the stage across human cancers. Lower (or Higher): the gene is associated with lower (or higher) stage (Spearman correlation test: $p<0.05)$, NS: No significant. (D) Correlation between the expression levels of $R C L 1$ and grade across human cancers. Lower (or Higher): the gene is associated with lower (or higher) stage (Spearman correlation: $p<0.05)$, NS: No significant. (E) Expression of Rcl1 in patients with HCC based on data from TCGA-LIHC and GEO datasets. p-value Significant Codes: * $<0.05$.

\section{Figure 2}

Low RCL 1 expression is correlated with worse prognosis in HCC patients from TCGA-LIHC dataset. (A) Kaplan-Meier curves analysis for the overall survival (OS) in TCGA-LIHC cohort using R software, patients were divided into two groups according to the median of Rcl1 expression. (B) Kaplan-Meier curves analysis for progression-free survival (PFS) in TCGA-LIHC cohort. (C) Forest plot of multivariate analysis of liver cancer OS using R software. (D) Forest plot of multivariate analysis of liver cancer PFS. AJCC American Joint Committee on Cancer.

\section{Figure 3}

Association between $R C L 1$ expression and clinicopathologic characteristics in the HCC cohorts. RCL 1 expression was lower in (A) female, (B) higher TNM stage, (C) higher T classification, (D) higher AFP levels, (E) vascular invasion in TCGA cohort, $(\mathbf{F})$ higher T classification, $(\mathbf{G}) \mathrm{HBV},(\mathrm{H})$ portal vein invasion, (I) hepatic vein invasion in ICGC cohort, and $(J)$ BCLC C stage, $(K)$ proliferation class, $(\mathbf{L})$ high AFP level, 
(M) vascular invasion, and the positive expression of $(\mathrm{N})$ phosphorylated Akt, $(\mathbf{0})$ phosphorylated RPS6,

(P) phosphorylated IGFR1 in GSE9843 cohort.

\section{Figure 4}

Correlation analyses between RCL 1 expression and immune infiltration levels in TCGA-LIHC cohort.

\section{Figure 5}

$R C L 1$ expression is strongly correlated with molecular- and immune-classifications of HCC. (A) Violin Plot of the RCL 1 expression in different molecular subtypes. (B) Violin Plot of the RCL 1 expression in different immune subtypes. C1 Wound healing, C2 IFN-y Dominant, C3 inflammatory, C4 Lymphocyte depleted, C6 TGF- $\beta$ Dominant. (C) Violin Plot visualizing the RCL 1 expression in wild-type and mutant TP53 gene. (D) Violin Plot visualizing the RCL 1 expression in wild-type and mutant CTNNB1 gene. (E) Violin Plot visualizing the RCL 1 expression in wild-type and mutant TERT gene. (F) Violin Plot visualizing the RCL 1 expression in wild-type and mutant IDH1 gene.

\section{Figure 6}

The Rcl1 expression in HCC cell lines. (A) Analysis of the Rcl1 mRNA expression in HCC cell lines. (B) Western blot analysis of the Rcl1 protein expression in HCC cell lines. (C) Immunofluorescence staining of the Rcl1 potein in HCC cell lines. p-value significance symbols: $*<0.05,{ }^{\star *}<0.01, \star \star \star<0.001$. Bar $=50 \mu m$

\section{Figure 7}

Rcl1 inhibits HCC cells proliferation, migration and invasion in vitro. (A, B) western blot analyses and (C, D) qRT-PCR assays of Rcl1 overexpression and knockdown in Huh-7 and Hep-3B cells. (E, F) CCK-8 assays of Huh-7 and Hep-3B cells after Rcl1 overexpression or knockdown with the corresponding negative controls. $(G, H)$ Transwell assays examined the migration and invasion activities of Rcl1 overexpression or knockdown with their corresponding negative controls. Data are presented as the mean \pm standard deviation from three independent experiments. ${ }^{\star} p<0.05,{ }^{*} p<0.01,{ }^{*} * \mathrm{p} p 0.001$. Bar $=100$ $\mu \mathrm{m}$.

\section{Figure 8}


Rcl1 is strongly correlated with cell cycle- and metabolism-related pathways. (A) Scatter plot of the significantly enriched Hallmark gene sets. Top 10 enriched pathways are shown in the figure. (B, C, D) The significantly enriched Gene Ontology annotations (molecular functions, biological processes, and cellular components). Top 20 enriched pathways are shown in the figure. (E, F) Cell cycle tests of Huh-7 and Hep$3 B$ cells after Rcl1 overexpression or knockdown with their corresponding controls. NS $>0.05,{ }^{*} p<0.05$, ${ }^{\star *} p<0.01, * \star * p<0.001$.

\section{Supplementary Files}

This is a list of supplementary files associated with this preprint. Click to download.

- SUPPLEMENTARFIGURERCL1CLINICALCHARACTERSTIC.tif 